\title{
ON THE EVOLUTION OF INFRASTRUCTURE SHARING IN MOBILE NETWORKS: A SURVEY
}

\author{
Lorela Cano $^{1}$, Antonio Capone ${ }^{2}$, Brunilde Sansò ${ }^{3}$ \\ ${ }^{1,2}$ Politecnico di Milano, Milan, Piazza Leonardo da Vinci, 32, 20133, Milano MI, Italy, ${ }^{3}$ Polytechnique Montréal, \\ 2500 Chemin de Polytechnique, Montréal, QC H3T 1J4, Canada,
}

NOTE: Corresponding author: Lorela Cano (lorela.cano@polimi.it)

\begin{abstract}
Infrastructure sharing for mobile networks has been a prolific research topic for more than three decades now. The key driver for Mobile Network Operators to share their network infrastructure is cost reduction. Spectrum sharing is often studied alongside infrastructure sharing although on its own it is a vast research topic outside the scope of this survey. Instead, in this survey we aim to provide a complete picture of infrastructure sharing both over time and in terms of research branches that have stemmed from it such as performance evaluation, resource management etc. We also put an emphasis on the relation between infrastructure sharing and the decoupling of infrastructure from services, wireless network virtualization and multi-tenancy in $5 G$ networks. Such a relation reflects the evolution of infrastructure sharing over time and how it has become a commercial reality in the context of $5 G$.
\end{abstract}

Keywords - 5G, infrastructure sharing, mobile networks, multi-tenancy, spectrum sharing, wireless network virtualization

\section{INTRODUCTION}

Infrastructure sharing in mobile networks is a multifaceted problem involving not only academic and industrial research entities but also national and international regulatory entities $[51,52,65,66]$, standardization bodies [1-4] and vendors [45,107]. In essence, infrastructure sharing in mobile networks is the shared use of existing or jointly deployed network infrastructure among multiple Mobile Network Operators (MNOs).

Based on which network elements (nodes) MNOs agree to/can share, there are two main types of sharing: passive and active, the latter comprising the former. Passive sharing (also referred to as site sharing or colocation [49]) implies the sharing of the site physical space and of the non-active elements on the site (such as shelter, cabinet, mast, etc. [49, 104]). Instead, active sharing extends to active elements of the Radio Access Network (RAN) (such as antennas, Base Transceiver Stations/Base Station Controller for 2G, Node B/Radio Network Controller for $3 \mathrm{G}$, eNode B for $4 \mathrm{G}$, and $\mathrm{gN}$ odeB for $5 \mathrm{G}$ ) and part of the core nodes (in fact, core node elements related to user billing and accounting are not shared).

The phenomenon of infrastructure sharing has disrupted the business model of a conventional MNO, that is, an MNO which is by itself responsible for (i) purchasing a spectrum license, (ii) deploying and managing the network infrastructure, (iii) tailoring services for their subscribers (e.g., voice, data, etc.) and (iv) handling their billing and accounting. The main reason for MNOs to share infrastructure is to divide the infrastructure cost among them and hence make their business more profitable. In these lines, infrastructure sharing has accompanied the technology migrations from $2 \mathrm{G}$ to $3 \mathrm{G}$ and from $3 \mathrm{G}$ to $4 \mathrm{G}$ due to the high upfront cost met by MNOs during these migrations. In turn, in 5G networks, infrastructure sharing, besides from being a means for cost-reduction, it is also an important pillar of the $5 \mathrm{G}$ architecture. Another paradigm strongly linked to infrastructure sharing is spectrum sharing. The need for spectrum sharing comes from spectrum being an intrinsically scarce resource, even more so in the context of $5 \mathrm{G}$, given its target throughputs. However, spectrum sharing alone is a really vast research topic and will be outside the scope of this survey unless combined with infrastructure sharing.

What's more, in this paper we will also address some literature on Wireless Network Virtualization (WNV) [91] and network slicing (enabling multi-tenancy) in the context of 5G [6], since both are based on infrastructure and spectrum sharing. Conversely, WNV and network slicing can be seen as enablers for infrastructure and spectrum sharing. Besides, another concept closely related to infrastructure and spectrum sharing is that of the decoupling of infrastructure from services, which was envisioned by some of the early literature on infrastructure sharing (see Section 2). The concept has been further carried out in the context of WNV and then in the context of network slicing. In fact, the different research efforts on introducing Software-Defined Networking (SDN), virtualization in general and Network Functions Virtualization (NFV) in particular into mobile networks seem to have converged into the $5 \mathrm{G}$ 
architecture as enablers for network slicing.

Infrastructure sharing in this broader sense has been a very prolific research topic over the last three decades. Samdanis et al. in [125] provide a compelling analysis of the path from infrastructure sharing to multi-tenancy. However, to the best of our knowledge, our survey is the first ${ }^{1}$ comprehensive study on how the infrastructure sharing topic in mobile networks has evolved over time, i.e., with the advent of the different mobile network generations, and which research branches have spurred from this topic. Reviewing this evolution is particularly important now that networking slicing is being introduced in $5 \mathrm{G}$ (from release 16 onwards) and operators are looking for models for sharing infrastructure costs and to invest more in new services and applications, collaborating with different players of vertical industrial sectors. Moreover, it is becoming clear to the telecommunications industry sector that some form of infrastructure sharing will be the common basis on which networks will be deployed in different countries and services will evolve and diversify, going beyond $5 \mathrm{G}$ and preparing the ground for the next generation.

This survey is organized in the following fashion. Due to the change in the nature of problems studied over time, we first make a broad chronological classification of the literature into early works and recent and up-todate works. For the latter, we further identify several research branches/categories. The overall picture of our classification is depicted in Fig. 1. An overview of the early works on the topic is provided in Section 2. Further, in Section 3, we focus on the more recent and upto-date works. Then in Section 4 we make a critical discussion of the research area related to infrastructure sharing and provide an outlook of future research directions. Finally, conclusions are drawn in Section 5.

For readers' ease, in Table 1 we provide the definitions of the acronyms and abbreviations used in the paper.

\section{EARLY WORKS}

$[16,49,55,70,115,118,142]$ are among the earliest articles on infrastructure sharing (combined at times also with spectrum sharing). With the exception of $[70]^{2}$, these articles have tended to:

- address technical issues of different sharing alternatives,

- assess the financial profitability through technoeconomic approaches,

\footnotetext{
${ }^{1}$ This survey is based on the PhD thesis of Lorela Cano [24].

${ }^{2}$ The study in [70] is an early work on the problem of scheduling users of multiple operators arising from the case when a $3 \mathrm{G}$, facility-based MNO hosts several Mobile Virtual Network Operators (MVNOs): the authors propose a non-pre-emptive priority queuing model for circuit-switched traffic applied through an admission control scheme.
}

\begin{tabular}{ll}
\hline 3GPP & $3^{\text {rd }}$ Generation Partnership Project \\
BS & Base Station \\
C-RAN & Cloud Radio Access Network \\
DCS & Digital Cellular System \\
EDGE & Enhanced Data rates for GSM Evolution \\
GERAN & GSM EDGE RAN \\
GSM & Global System for Mobile communications \\
IaaS & Infrastructure as a Service \\
InP & Infrastructure Provider \\
IoT & Internet of Things \\
IP & Integer Programming \\
JV & Joint Venture \\
MLFG & Multi-Leader-Follower Game \\
mmWave & millimiter Wave \\
MNO & Mobile Network Operator \\
MVNO & Mobile Virtual Network Operator \\
NaaS & Network as a Service \\
NFV & Network Functions Virtualization \\
NSP & Network Service Provider \\
OTT & Over The Top \\
PRB & Physical Resource Block \\
QoS & Quality of Service \\
RAN & Radio Access Network \\
RRH & Remote Radio Head \\
SaaS & Software as a Service \\
SDN & Software-Defined Networking \\
SINR & Signal-to-Interference-plus-Noise Ratio \\
SLA & Service Level Agreement \\
SP & Service Provider \\
UMTS & Universal Mobile Telecommunications System \\
VNO & Virtual Network Operator \\
VO & Virtual Operator \\
W-CDMA & Wideband Code Division Multiple Access \\
WNV & Wireless Network Virtualization \\
xG & x th mobile network Generation \\
\hline &
\end{tabular}

Table 1 - Definitions of acronyms and abbreviations

- state regulatory standpoints and provide guidelines for the latter and

- conceive new paradigms for the mobile market.

In [118], which dates back to 1994, Ramsdale states that national roaming ${ }^{3}$ is part of the specifications of the Digital Cellular System at $1800 \mathrm{MHz}$ (DCS 1800), unlike the Global System for Mobile Communications at 900 $\mathrm{MHz}$ (GSM 900), which supported international roaming only. National roaming was introduced in the DCS 1800 to improve coverage due to smaller cell sizes at $1800 \mathrm{MHz}$ (as opposed to $900 \mathrm{MHz}$ ).

Instead, the work in [55] shows the positive impact of infrastructure sharing in financial terms for the Universal Mobile Telecommunications Systems (UMTS), especially for lowly populated areas in which network deployment is dictated by coverage instead of capacity.

\footnotetext{
${ }^{3}$ National roaming is an infrastructure sharing alternative that allows users of an operator which does not provide coverage in certain areas of a country to be served by the network of another operator of that country covering such areas.
} 


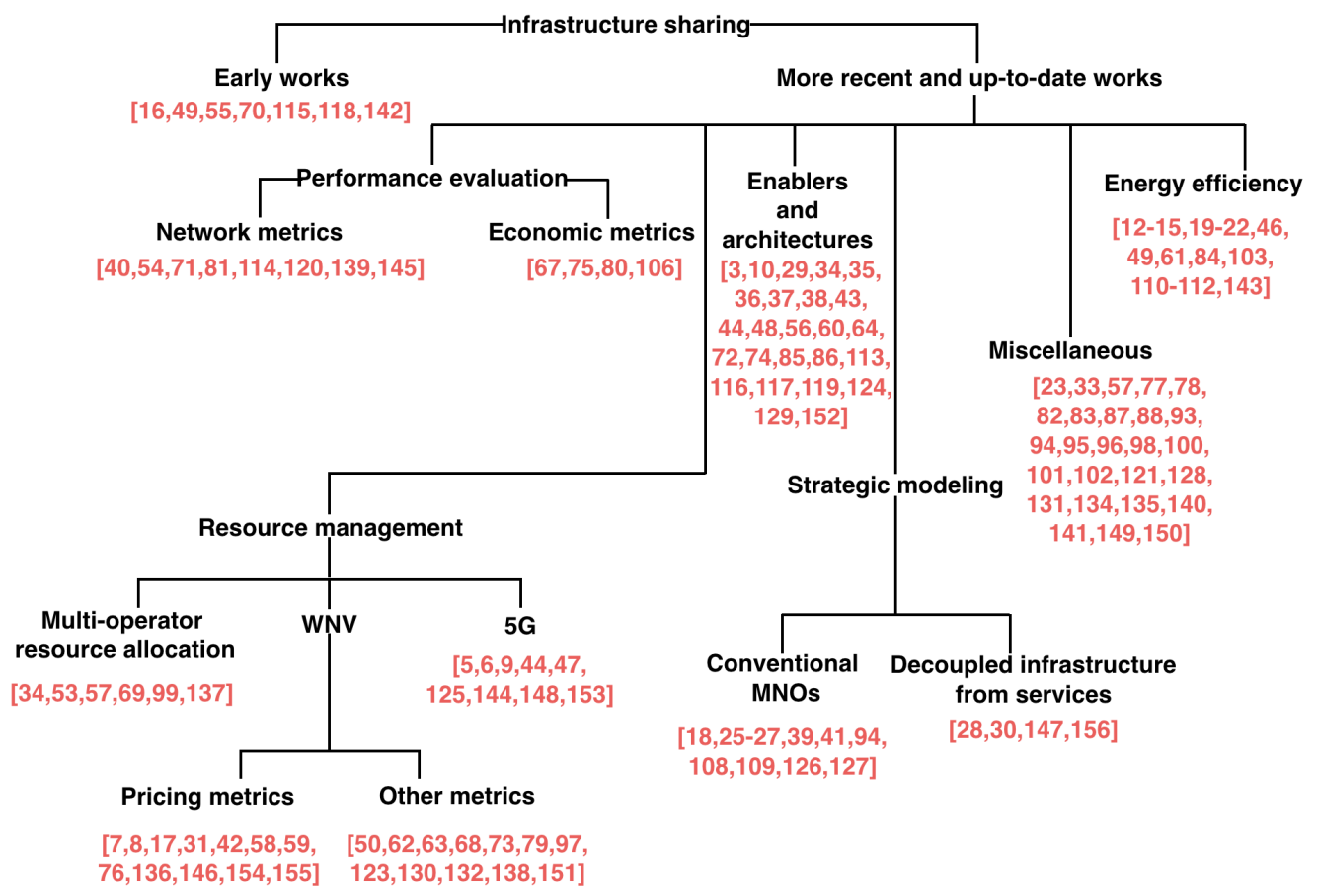

Fig. 1 - Literature classification map

In turn, MVNOs are suggested as a means to monetize spare resources of an MNO.

Park et al. in [115] discuss issues faced by MNOs worldwide when deploying Wideband Code Division Multiple Access (W-CDMA) and propose spectrum trading and infrastructure sharing as means to accelerate the deployment of W-CDMA. However, they emphasize that such means should be cautiously treated by regulators.

The study in [49] proposes a spreadsheet-based financial model to estimate the economic profitability of multiple sharing alternatives and shows that cost can be further reduced if the network operations are outsourced or a joint venture is created.

The authors in [142] discuss technical aspects concerning the infrastructure sharing alternatives at the time; they also anticipate two crucial paradigms: (i) dynamic spectrum trading and (ii) the decoupling of the network infrastructure from services, enabled by infrastructure sharing. It is worth noticing that both these paradigms are ongoing research topics even nowadays. Similarly, according to [16], the advantages of network sharing go beyond cost reduction: based on the product life cycle model, the authors suggest that, under an appropriate regulatory framework, network sharing can steer the monolithic mobile networks industry toward the decoupling of the network infrastructure from services for end users. In other words, based on [142] and [16] infrastructure sharing would lead to new stakeholders such as network/infrastructure providers ( $\mathrm{InPs}$ ) and service providers (SPs) which were expected to emerge in the mobile market, the former being responsible for network planning, deployment and management while the latter for dealing only with the development of novel services (possibly specialized and targeting specific market segments [16]).

When analyzing these early works on infrastructure sharing in mobile networks, we have to consider the specific technical limitations that have constrained the approaches for $2 \mathrm{G}, 3 \mathrm{G}$ and partially $4 \mathrm{G}$ network, to some aspects of the problem only. In particular, being the spectrum one of the most important assets of a mobile network and being it easily shared among physically separated networks, it has been widely studied considering the locality of interference generated and the re-usability in different geographical areas.

As far as the physical infrastructure is concerned, the first works on sharing have focused on economic aspects and market regulation policies associated to the introduction of MVNOs. However, the main limitation of these approaches was due to the mobile technology that prevented a significant service and performance differentiation among users of MVNOs and MNOs. Therefore, sharing policies had to be based on other objectives such as cooperative coverage of low population areas and cost sharing of radio towers.

As mentioned above, the key aspect that we take from these works is the decoupling of network services from the infrastructure that provides them. Only recently, however, this concept has become fully exploitable thanks to the network virtualization technologies that allow a fine grain differentiation of the network behavior with respect to different applications and groups of 
users. This radical change of the technology scenario, mainly due to the new architectural solutions and service definition of $5 \mathrm{G}$, did not cancel the main issues analyzed by the early works on infrastructure sharing, such as the economic aspects of cost sharing and their relation with resource allocation or partitioning.

\section{MORE RECENT AND UP-TO- DATE WORKS}

In the more recent and up-to-date literature, there is a tendency to address specific problems, e.g., the problem of resource management, for specific sharing scenarios, e.g., infrastructure and spectrum sharing at the RAN. There are at least two ways to go about the classification of this literature, one being problem-centric and the other being methodology-centric. We have opted for the first one in order to highlight the fact that there are many aspects to infrastructure sharing and hence provide the reader with the bigger picture on the topic. Methodology details are discussed only when deemed necessary.

Under the problem-centric classification, we have identified the following research branches/categories for the revised articles: (i) performance evaluation, (ii) resource management, (iii) enablers and architectures, (iv) energy efficiency, (v) strategic modeling and (vi) miscellaneous.

It is worth pointing out that some of the articles may fit in more than one category, but for each such article, we have opted for a single category, the one we believe is the most salient.

\subsection{Performance evaluation}

Several authors have addressed the gains of particular infrastructure and/or spectrum sharing scenarios in terms of network performance metrics, such as throughput, coverage probability etc. (see e.g., [71, 114, 139, 145]) and/or economic ones such as CAPEX/OPEX reduction (see e.g., $[67,75,80,106])$. The common approach is to benchmark such scenarios against the baseline case when no sharing takes place and the involved MNOs build individual networks instead. Methodologywise, both theoretical, mainly stochastic geometry analysis (see e.g., [54,71,81,145]), and simulation approaches (see e.g., $[40,114,120]$ ) have been adopted. For instance, the work in [114] proposes a virtualized architecture to enable two types of spectrum sharing other than the classical one and capacity sharing (national roaming) and compares the different sharing alternatives with no sharing case. The performance metrics considered in [114] are the sector load and packet drop probability.

The authors in [40] analyse how the time and space correlation of the MNO individual traffic loads impacts the gains of infrastructure sharing in the case when MNOs decide to pool together their respective networks. Kibilda et al. [81] resort to stochastic geometry to calculate the gains of sharing for the cases of infrastructure and/or spectrum pooling. Their key finding is that the infrastructure and spectrum sharing gains do not sum up when combined since full sharing (infrastructure+spectrum) introduces a trade-off between the data rate and coverage.

As $5 \mathrm{G}$ is expected to make use of the millimeter wave (mmWave) frequencies [11], the gains of infrastructure and/or spectrum in these frequencies have become the object of several recent articles. For instance, Gupta et al. in [54] provide a stochastic geometry-based theoretical analysis on the gains of spectrum sharing using a simplified antenna and channel model for the mmWave frequency range. In particular, in [54] it is shown how narrow beams are key for spectrum sharing in the mmWaves. A very similar investigation to [81] is carried by Rebato et al. in [120] for mmWaves; the authors highlight the impact of the channel model accuracy when carrying out a quantitative analysis of the sharing gains. The recent work in [71] also addresses infrastructure and spectrum sharing at mmWaves and it resorts to stochastic geometry to derive the probability of Signal-to-Interference-plus-Noise Ratio (SINR) coverage as a performance metric.

In Table 2 we provide a visual overview of the classification of the different articles that were included in the performance evaluation category. As can be seen from the table, methodologically-wise, the authors use mainly stochastic geometry, simulation and optimization. The other method found was empirical analysis. With respect to the type of measures used to evaluate performance, we can see that network measures are fairly diverse: even though most work in this category deals with physical layer measures such as SINR, networking measures such as traffic load, sector overload or packet drop probability are also considered. Not surprisingly, less diversity can be found in the economic measures' category.

\subsection{Resource management}

Problems of resource management arise whenever infrastructure sharing is combined with spectrum sharing, as users of multiple MNOs/MVNOs have to be assigned resources from a shared pool.

Several studies $([34,53,99,137])$ have proposed algorithms for a multi-operator scheduler, namely when users of multiple MNOs have to be scheduled in the finite resources available in a shared Base Station (BS). Assuming MNOs agree a priori on the resource shares, i.e., how to split the available BS resources among them, the work in [137] adopts the concept of Generalized Processor Sharing for a multi-operator scheduler. For the same setting, Malanchini et al. [99] explore the trade- 


\begin{tabular}{|c|c|c|c|c|c|}
\hline \multicolumn{2}{|c|}{ Classification } & Stochastic Geometry & Simulation & Optimization & Other \\
\hline \multirow{7}{*}{$\begin{array}{l}\text { Network } \\
\text { Measures }\end{array}$} & traffic load & & [40] & [67] & \\
\hline & SINR & [54], [71] & {$[120]$} & {$[80]$} & \\
\hline & $\begin{array}{l}\text { SINR coverage } \\
\text { probability }\end{array}$ & {$[71],[81]$} & & & \\
\hline & Throughput & & & [67] & \\
\hline & User rate & [81], [145] & & & \\
\hline & Sector overload & & [114] & & \\
\hline & $\begin{array}{l}\text { Packet drop proba- } \\
\text { bility }\end{array}$ & & [114] & & \\
\hline \multirow{4}{*}{$\begin{array}{l}\text { Economic } \\
\text { Measures }\end{array}$} & Revenue & & [75] & & \\
\hline & CAPEX & & [75] & & [106] \\
\hline & OPEX & & [75] & & \\
\hline & Miscellaneous & & [75] & {$[67],[80]$} & \\
\hline
\end{tabular}

Table 2 - Performance evaluation classification

off between satisfying the resource shares and improving the overall (system) spectral efficiency when the agreed resource shares are violated in a controlled fashion. The work in [53] considers a global scheduler taking decisions for clusters of BSs and therefore scheduling users of multiple MNOs over a 3D time-frequency-space resource grid. In [53] scheduling is performed with the objective of maximizing the overall system utility. The authors in [34] propose a BS virtualization scheme which performs scheduling in two levels, namely, among MNOs, and for each MNO, among its user flows. Hew et al. in [57] consider a network shared by multiple MNOs, each of them serving both a set of end users and a set of MVNOs. In this context, the problem of resource allocation is tackled in two steps: first, the resource sharing among MNOs, and then the resource sharing among the users and the MVNOs of each MNO, where the resource sharing at each step is modeled as a bargaining problem. The study in [69] suggests an algorithm that fairly allocates the shared radio resources among MNOs. In [105] the authors propose Remote Radio Head (RRH) assignment algorithms for an SDN-based Cloud Radio Access Network (C-RAN) shared by multiple MNOs.

Concerning WNV, the problem of resource management is crucial in the interaction between an InP and its SPs. In the context of this paper, an InP is an entity which is responsible for the infrastructure deployment, management and operation and does not serve end users directly whereas an SP is an entity which does not have any resources of its own but purchases or rents resources from an InP to provision services for its end users. It is worth noticing that the terminology concerning the SP varies across different articles: such an entity is also referred to as a Virtual Operator (VO), a Virtual Network Operator (VNO) or a Mobile Virtual Network Operator (MVNO). Also notice that the conventional MVNO obtains resources from an MNO which serves end users of its own, unlike the InP. The key difference lies in the fact that a conventional MVNO competes with its MNO, while there is no such competition between an $\mathrm{InP}$ and its SPs/VOs/VNOs/MVNOs. In these lines, some articles tend to "misuse" the term InP when they consider the InP to provide services also to end users. Additionally an InP is also referred to as a Network Service Provider (NSP). Moreover, the work in [91] envisions three different types of stakeholders in line with the ones in the cloud computing domain, i.e., the InP providing Infrastructure as a Service (IaaS), the MVNO providing Network as a Service (NaaS) and the SP providing Software as a Service (SaaS). For instance, in [89] the authors address a scenario in which there are multiple InPs, a single MVNO and multiple SPs where the MVNO acts as a reseller of resources from InPs to SPs. It should also be noted that the terms slicing and slice are also misused in some articles in non-5G contexts, in the sense that, such articles do not consider problem instances that account for $5 \mathrm{G}$ service requirements.

There is a large body of literature on resource managements concerning InPs and SPs in the context of WNV. The vast majority of articles in this literature considers a single InP and multiple SPs (see e.g., $[7,8,17,31,42,50,58,59,62,63,68,73,76,79,97,123,130$, $132,133,136,138,146,151,154,155])$. However, there are exceptions: e.g., the work in [32] considers a single InP and a single VNO which serves multiple users through an SDN-based virtualized network provided by the InP. The VNO faces the problem of scheduling its users, each characterized by a maximum delay over a finite time period, through resources rented by the $\mathrm{InP}$ with the objective of minimizing the payments made to the InP for the rented resources. There are also articles which consider both multiple InPs and multiple SPs (and few other variations with multiple InPs) which however are more pertinent to Section 3.5 hence discussed therein.

As for the literature on a single InP and multiple SPs, 
it can be broadly classified into two groups based on whether the resource management is driven by pricing $([7,8,17,31,42,58,59,76,136,146,154,155])$ or not $([50,62,63,68,73,79,97,123,130,132,133,138,151])$. For instance, Ho et al. in [58] consider the case when there is a single InP serving multiple MVNOs, each characterized by a fixed number of users and a Service Level Agreement (SLA) given in terms of a minimum resource requirement and a maximum aggregate rate (over all its users). The InP has to decide how to price and allocate its available BS resources among all users of all MVNOs so as to maximize its profit while guaranteeing the SLA of each MVNO. In this work MVNOs are also self-interested as the goal of each MVNO is to maximize its own profit given by the difference between the total rate obtained from resources allocated by the InP and their cost. The problem is then modeled as a one-leader multi-follower variant of the Stackelberg game with the InP being the leader and each MVNO being a follower. Instead, Kamel et al. in [73] address a scheduling problem over one time frame which is modeled through mathematical programming. In details, there is a single InP and a set of VOs, each having a fixed number of users and a minimum resource requirement (total Physical Resource Blocks (PRBs) over the time frame). The InP has to decide to which user to assign each PRB and the amount of power to allocate to each PRB so as to maximize the total rate over the time frame while satisfying the maximum power constraint, the minimum resource requirement of each $\mathrm{VO}$ and a $\mathrm{VO}$-specific proportional fairness constraint for cell-center and cell-edge users.

In $5 \mathrm{G}$, the problem of resource management reemerges in the context of multi-tenancy and its enabler, network slicing $([5,6])$. Tenants (such as MVNOs, Over The Top (OTT) providers and vertical industries) have distinct requirements to support their services which have to be translated into appropriate network resources. It is worth noting that network slicing does not involve only the RAN segment but it can be end-to-end. However, the problem of resource management at the RAN segment has brought about a significant amount of attention from the research community due to the intrinsically complex nature of the radio (wireless) access. For instance, the authors in [125] propose the "5G Network Slice Broker", a centralized scheduler based on the $3^{\text {rd }}$ Generation Partnership Project (3GPP) specifications for network sharing. The proposed scheduler has a global view of the shared network and applies admission control and resource allocation, translating the tenants' request, with given SLAs, into available network resources. Other examples on resource management at the RAN in the context of multi-tenancy/network slicing are given in $[9,44,47,122,144,148,153]$.

Summarizing, the Resource Management category is a very rich part of the infrastructure sharing literature. Within this category, we have identified three sub- categories:

1. partitioning and allocation of resources shared by multiple operators,

2. the literature on Wireless Network Virtualization that mostly deals with the sharing of resources between a single InP and multiple SPs; this subcategory, can be further subdivided into:

(a) articles that base their modelling on pricing issues and

(b) articles that base their modelling on other issues, such as performance metrics, and

3. a large body of $5 \mathrm{G}$ literature that deals with resource management and network slicing.

\subsection{Enablers and architectures}

Although the different alternatives for infrastructure and spectrum sharing can be financially attractive for MNOs, they where not always supported by the $3 \mathrm{GPP}$ specifications; in fact, while a basic type of network sharing was supported as of Release 5, there was no support for more involved network sharing scenarios for the 3GPP GSM EDGE ${ }^{4}$ RAN (GERAN) prior to Release $10([3])$.

Standardization apart, the research community has largely contributed on the topics of enabling network sharing, e.g., through novel architectures. While passive sharing (i.e., site/tower sharing) is the simplest network sharing alternative to implement, the different types of active sharing demand architectural changes in mobile networks e.g., to guarantee the isolation of the involved MNOs in terms of their private information in order to avoid harming competition, or they demand changes at the protocol stack level to implement the novel resource management algorithms etc. According to [64], radio resource management should be delegated to a third party provider to ensure isolation and therefore not to interfere with competition. In [56] the authors introduce AppRAN which relies on a centralized scheduler to perform application-level resource allocation for a shared RAN.

In particular, different flavors of virtualization have been widely considered by the research community as candidate enablers for network sharing. For instance, the virtualized network architecture proposed in [60] can support network sharing. Other papers that resort to virtualization are e.g., $[10,34,72,117,152]$. In particular, the authors of [38] and of [43] propose the "Network without Borders", namely the virtualized pool of (heterogeneous) wireless resources for which infrastructure and spectrum pooling are essential. Costanzo et al. in [37] suggest an architecture for $4 \mathrm{G}$ RAN sharing based on SDN and NFV.

\footnotetext{
${ }^{4}$ Enhanced Data rates for GSM Evolution
} 
In the context of enabling network slicing in 5G networks, there is a myriad of papers that propose architectures or test prototypes based on (i) NFV and/or SDN (see e.g, $[35,36,85,86,113,119]$ ), (ii) changes to the RAN protocol stack (see e.g., $[48,116,124]$ ), or (iii) using features of the new $5 \mathrm{G}$ radio ([44]) etc. In particular, the work in [29] proposes an architecture to support network slicing in ultra-dense networks, the one in [74] presents an architecture that supports Internet Of Things (IoT) slices whereas the one in [129] dwells on combining $3 \mathrm{GPP}$ specifications for $5 \mathrm{G}$ with NFV.

\subsection{Energy efficiency}

Infrastructure and spectrum sharing allow to reduce the energy-consumption OPEX cost particularly in cases when the aggregated network resources (infrastructure and/or spectrum) are redundant. For instance, in rural areas where capacity is not an issue, MNOs can decommission a subset of the aggregated BSs and/or operate at a subset of the aggregated frequency carriers [49], which reduces the energy consumption and (indirectly) the environmental impact. In these lines, since MNOs dimension their networks based on the peakload traffic predictions, there is intrinsically resource redundancy during the off-peak periods in their individual networks. Consequently, MNOs can agree to roam users of each other during the off-peak periods, e.g., overnight, and switch off a subset of their BSs (see e.g., $[13,21])$. While the vast majority of infrastructure (and spectrum) sharing problems revolve around economic and technical aspects, some papers (see e.g., [12-15, 19-22,46,61,84,103,110-112,143]) have taken an energy-efficiency/green networking perspective.

\subsection{Strategic modeling}

This branch consists of articles that deal with decisionmaking problems such as MNOs deciding whether to enter a sharing agreement or not, SPs selecting InPs from which to obtain resources etc. In these lines we can further split this category into two subcategories: (i) infrastructure sharing among conventional MNOs and (ii) infrastructure sharing for decoupled infrastructure from services (involving InPs and SPs etc.). Such articles naturally resort to mathematical programming and to game theory in particular when the involved actors are assumed rational, self-interested and payoff-maximizing entities.

\subsubsection{Infrastructure sharing among conven- tional MNOs}

The following articles concern either greenfield deployment of shared networks $[18,25-27,108,109,127]$ or the case when shared networks are created by pooling together the existing network infrastructure of at least two MNOs [39, 41, 94, 126].
Blogowski et al. in [18] deal with the particular scenario when two MNOs have to deploy BSs over a given set of candidate sites. For each site, each MNO has to decide whether to install a BS or not; in the former case, if both MNOs decide to install a BS, it is assumed that it is profitable for both to install a single shared BS. The problem is formulated as a non-cooperative game where the payoff of each player (MNO) is given by its total profit (revenues - cost), calculated over all BSs. It is assumed that each site can serve a given (arbitrary) number of users, e.g., those under its coverage area, which means there are no capacity constraints associated with the sites. Instead, coverage constraints are present and they are expressed as a minimum percentage of users to be served by each MNO (a common constraint associated for spectrum licensees). When the coverage constraint is absent, MNOs can decide independently for each site. Otherwise, the game is no longer separable. The authors describe the propriety of the Nash equilibria of the game for different relationships of the payoff matrix (i.e., by establishing relations between the payoffs obtained under different strategy profiles) and also suggest a centralized solution which Pareto dominates all Nash Equilibria.

$[108,109,127]$ address the problem of infrastructure and spectrum sharing arising when a set of MNOs, each with a given number of users (market share) and own spectrum license, plan a greenfield Long-Term Evolution (LTE) deployment. The strategic problem of coalition formation, namely, which subsets of MNOs voluntarily sign long-term infrastructure and spectrum sharing agreements, is modeled by means of non-cooperative game theory. We address a very similar problem to $[108,109,127]$ in $[25,26]$ resorting to cooperative game theory in [26] and non-cooperative game theory in [25]. Unlike in $[108,109,127]$, in $[25,26]$ we (i) account for both the technical and economic aspects of sharing reflected in the payoff function definition and (ii) do not split the shared infrastructure cost among MNOs a priori; how these cost are split is an outcome of the model (game). In turn in [27], we address a similar scenario to $[25,26]$ but without spectrum pooling. Moreover, in [27] we consider two different cases deriving from two different perspectives, the one of a regulatory entity favoring the users and the MNOs' perspective as profit-maximizers. We model the former case through Mixed Integer Linear Programming and the latter through cooperative game theory.

The authors in [41] consider the case when a set of MNOs agrees to pool together their current individual RAN networks but make joint decisions for future decommissions, network expansion and upgrades of their shared network; a greedy procedure is proposed to solve the multi-period network planning.

Similarly to the "sale-leaseback" approach of Tower Companies (see e.g., [90]), the work in [39] assumes a set 
of self-interested MNOs decide to pool together their respective network infrastructures and create a Joint Venture $(\mathrm{JV})$, responsible for managing their shared network. In turn, MNOs will leaseback network capacity from the JV. The authors propose a Stackelberg game to determine the shares MNOs obtain from the JV and the prices set by the JV to the MNOs and by the MNOs to their respective users.

Notably, the user perspective is considered in [94], which investigates the problem of user-to-BS association when multiple MNOs decide to pool together their respective network infrastructures. The authors propose a noncooperative game to model the problem of each user selecting its serving BS from the shared pool, independently, so that its individual data rate is maximized.

The work in [126] represents a fresh take on infrastructure sharing. Its authors consider a set of MNOs with individual but overlapping infrastructures (BSs) and individual spectrum licenses; in this setting one of the MNOs (the buyer) can purchase the use of BSs of the other MNOs (the sellers) for serving its own users at its own licensed spectrum. The buyer MNO evaluates whether it can provide a given (Quality of Service) QoS to its own users through its own infrastructure by increasing the transmission power of its BSs or by purchasing BSs from the seller MNOs. In the latter case, the buyer MNO has to decide from which seller MNOs to buy from and what fraction of their BSs to purchase so as to minimize its expenditures while satisfying the QoS of its users. In turn, the seller MNOs have to decide the fraction of their own BSs to sell so as to maximize their profit (payment from the buyer MNO minus cost of sold BSs) where the competition in quantity among the seller MNOs is modeled as a Cournot market.

\subsubsection{Infrastructure sharing for decoupled in- frastructure from services}

We remind the reader that we have discussed the varying terminology used across different articles related to the infrastructure sharing for decoupled infrastructure from services in Section 3.2 and that we have maintained the authors' terminology for the considered stakeholders when describing their articles and, when necessary, we provide clarifications on how they compare to our definitions of InPs and SPs.

It is worth pointing out that, across the different articles very distinct mathematical approaches have been used to study the interaction among InPs and SPs.

Rather exceptionally, the study in [30] tackles the interaction among InPs and MVNOs (analogous to SPs) from the MVNO perspective. In fact, the authors in [30] consider multiple InPs but a single MVNO and propose a model based on contract theory in which the MVNO acts as the employer whereas the InPs as employees.

Instead, Wei et al. in [147] take a centralized approach. Specifically, the work in [147] considers multiple InPs and multiple VNOs (analogous to SPs) in the context of WNV. Here, each InP has a given set of users of its own; resources allocated to its own users are referred to as local slices and the total rate across the local slices should be above a given minimum for each InP. Instead, resources allocated to users of an MVNO are referred to as foreign slices. Each $\mathrm{InP}$ is characterized by a given bandwidth (number of subchannels) and power budget for the downlink of a BS. The problem consists in determining the number of subchannels and amount of power to allocate to each slice by each InP. The objective is to maximize the total rate across all slices while satisfying the bandwidth and power constraints and the minimum rate requirement for the local slices of each InP. Consequently, the problem is formulated by means of an Integer Programming (IP) model. In this model an MVNO can be simultaneously served by multiple InPs, likewise an InP can simultaneously serve multiple MVNOs.

The authors in [156] propose a hierarchical (two layer) combinatorial auction to model the interactions among multiple InPs, multiple MVNOs (analogous to SPs), and multiple end users concerning the resource allocation at the BS level (the resources here being transmission power, number of channels and number of antennas).

In [28] we propose a novel framework based on a MultiLeader-Follower Game (MLFG) to study the technoeconomic interactions among multiple InPs and multiple SPs in a $5 \mathrm{G}$ context.

Table 3 summarizes the main issues that are considered in this subcategory: what are the actors that intervene in the infrastructure sharing scheme and what is the modelling and mathematical approach that is taken in each case.

\begin{tabular}{ccc}
\hline Article & Actors & Approach \\
\hline$[30]$ & many InPs - one SP & contract theory \\
{$[147]$} & many InPs - many SPs & IP model \\
{$[156]$} & many InPs - many SPs & auction theory \\
{$[28]$} & many InPs - many SPs & MLFG \\
\hline
\end{tabular}

Table 3 - Infrastructure sharing for decoupled infrastructure from services

\subsection{Miscellaneous}

\subsubsection{Infrastructure sharing for mobile net- work segments other than the access}

Infrastructure sharing and multi-tenancy can also be applied to specific segments of a mobile network other than the access. For instance, the studies in $[23,98,128,140$, 141] address sharing of the backhaul network whereas 
the one in [83] deals with the sharing of the core network.

\subsubsection{Infrastructure sharing among different types of networks}

In the following paragraph we provide some examples of heterogeneous infrastructure sharing. The work in [78] studies sharing among different Radio Access Technologies (RATs), the one in [102] addresses sharing between LTE femtocells and Wi-Fi hotspots whereas the one in [100] investigates $3 \mathrm{G}$ offloading over $\mathrm{Wi}-\mathrm{Fi}$. Kibilda et al. [82] deal with sharing among MNOs and OTTs. In [101] the authors propose a RAN architecture for both infrastructure and spectrum sharing between the MNOs and safety services. Instead the study in [95] concerns infrastructure sharing between mobile services and smart grid utilities or intelligent transportation services. Lin et al. in [93] address backhaul sharing among mobile networks and fixed networks whereas Simo-Reigadas et al. in [131] suggest exploiting the community infrastructure as backhaul for $3 \mathrm{G}$.

\subsubsection{Infrastructure sharing for networks other than mobile}

The concept of infrastructure sharing is not exclusive to mobile networks. In fact, it has been applied to fixed access networks and problems related to the latter have been recently addressed in the literature (see e.g., [33] and [77]). Apart from fixed access networks, infrastructure sharing has also been proposed for $\mathrm{Wi}-\mathrm{Fi}$ networks, e.g., in [121].

\subsubsection{Spectrum sharing}

As previously stated, the overall literature on the different types of spectrum sharing alone (i.e., not combined with infrastructure sharing) is per se very vast. Unsurprisingly, as spectrum is a scarce resource for the MNOs, many papers within this literature resort to different game theory models (see e.g., $[94,135,149,150]$ ).

\subsubsection{MVNO business model}

The relation among the MNO, its MVNO(s) and the end users has been largely addressed through game theory as well (see e.g., [57, 87, 88, 96, 134]).

\section{DISCUSSION AND OUTLOOK}

The large body of literature analyzed in this survey and the impact it has had over the years on the standardization of mobile technologies and the practices adopted around the world, testify that mobile networks are important infrastructures with high costs which can be shared in some scenarios in order to offer better and more convenient services to end users. Also, in terms of regulatory strategies adopted by national authorities in different countries, that over the years have favoured the introduction of MVNOs and roaming mechanisms, we can observe that the wide service availability at reasonable prices has been considered particularly important also with respect to natural market competition.

With the arrival of $5 \mathrm{G}$, we are observing a renewed interest in sharing strategies due to the specific virtualization technology available and the standardization of network slicing. For the first time, the dynamic allocation of network resources and the service specialization on different slices, allow serving different groups of users according to different quality levels. This will likely generate the interest of new players specialized in vertical application domains, in order to take the role of slice tenants and sharing the resources of the network infrastructures with others. Even extreme scenarios where communications resources are traded in real time on automated markets are now possible [92], like it already happens in other sectors like energy networks. With this regard, the use of the large toolbox created by research over several years will certainly be an important asset to be used to shape sharing and trading instruments.

There are however, other important evolution trends of the technology that will probably influence the sharing methods beyond $5 \mathrm{G}$. We want to mention here two of them that we believe are particularly relevant:

- the extreme distribution of access infrastructure with the so-called smart radio environments, and

- the full virtualization of connectivity in open and cloud-based architectures.

As for the smart radio environments, they consider the introduction of new equipment at the radio interface of mobile networks, which includes smart repeaters with large antenna arrays and controllable reflective surfaces. This kind of evolution trend is making clear that in the future the deployment of multiple physical infrastructures by different operators will become more and more difficult and the focus of MNO attention will shift from optimizing their own network to that of efficiently controlling the resources leased from the common infrastructure.

While the full virtualization of connectivity will be complete relatively soon, the evolution started years ago with the separation of the network logic from the pure transmission technology. There are here however, important novelties associated with open source approaches like Open RAN that are becoming popular and that are fostering the transition to cloud-based solutions where the value for service providers will be in designing and implementing advanced services based on the effective use of transmission resources. 


\section{CONCLUSION}

Infrastructure sharing in mobile networks has been a pervasive research topic over the last three decades and has produced a significant body of work.

One interesting takeaway from this survey is that while researchers sought enabling technologies to materialize infrastructure sharing in $3 \mathrm{G}$ and $4 \mathrm{G}$ networks, in $5 \mathrm{G}$ networks instead, infrastructure sharing became an important pillar of the $5 \mathrm{G}$ architecture which means that in turn $5 \mathrm{G}$ enables infrastructure sharing from a business point of view. In these lines one can easily argue the presence of infrastructure sharing also in future networks.

As for the mobile ecosystem, it is worth noting that the concepts of decoupling infrastructure from services and dynamic spectrum trading have been anticipated in the literature over two decades ago but they have come into being only recently (mainly in 5G networks).

Of the several research branches within the infrastructure and spectrum sharing topics, resource management in the context of resource sharing has been and will be one of the most active research branches given the current and future need for dynamic resource sharing.

\section{REFERENCES}

[1] 3GPP. TR 22.852, v13.1.0, Study on Radio Access Network (RAN) sharing enhancements (Release 13), September 2014.

[2] 3GPP. TR 22.951, v15.0.0, Service aspects and requirements for network sharing (Release 15), July 2018.

[3] 3GPP. TS 23.251, v15.1.0, Network sharing; Architecture and functional descriptions (Release 15), September 2018.

[4] 3GPP. TS 32.130, v15.0.0, Telecommunication management; Network sharing; Concepts and requirements (Release 15), June 2018.

[5] 5G NORMA. Deliverable D3.3, 5G NORMA network architecture - final report, October 2017.

[6] 5GPPP Architecture Working Group. View on 5G architecture, December 2017.

[7] H. Ahmadi, I. Macaluso, I. Gomez, L. DaSilva, and L. Doyle. Virtualization of spatial streams for enhanced spectrum sharing. In 2016 IEEE Global Communications Conference (GLOBECOM), pages 1-6. IEEE, 2016.

[8] Ö. U. Akgül, I. Malanchini, V. Suryaprakash, and A. Capone. Dynamic resource allocation and pricing for shared radio access infrastructure. In 2017 IEEE International Conference on Communications (ICC), pages 1-7. IEEE, 2017.
[9] O. Al-Khatib, W. Hardjawana, and B. Vucetic. Spectrum sharing in multi-tenant $5 \mathrm{G}$ cellular networks: Modeling and planning. IEEE Access, 7:1602-1616, 2019.

[10] L. Anchora, M. Mezzavilla, L. Badia, and M. Zorzi. A performance evaluation tool for spectrum sharing in multi-operator LTE networks. Computer Communications, 35(18):2218-2226, November 2012.

[11] J. G. Andrews, S. Buzzi, W. Choi, S. V. Hanly, A. Lozano, A. C. Soong, and J. C. Zhang. What will $5 \mathrm{~g}$ be? IEEE Journal on selected areas in communications, 32(6):1065-1082, 2014.

[12] C. Anglano, M. Guazzone, and M. Sereno. Maximizing profit in green cellular networks through collaborative games. Computer Networks, 75:260-275, 2014.

[13] A. Antonopoulos, E. Kartsakli, A. Bousia, L. Alonso, and C. Verikoukis. Energy-efficient infrastructure sharing in multi-operator mobile networks. IEEE Communications Magazine, 53(5):242-249, 2015.

[14] O. Aydin, E. A. Jorswieck, D. Aziz, and A. Zappone. Energy-spectral efficiency tradeoffs in $5 \mathrm{G}$ multi-operator networks with heterogeneous constraints. IEEE Transactions on Wireless Communications, 16(9):5869-5881, 2017.

[15] Y. Bao, J. Wu, S. Zhou, and Z. Niu. Bayesian mechanism based inter-operator base station sharing for energy saving. In Communications (ICC), 2015 IEEE International Conference on, pages 49-54. IEEE, 2015.

[16] C. Beckman and G. Smith. Shared networks: Making wireless communication affordable. IEEE Wireless Communications, 12(2):78-85, April 2005.

[17] D. Bega, M. Gramaglia, A. Banchs, V. Sciancalepore, K. Samdanis, and X. Costa-Perez. Optimising $5 \mathrm{G}$ infrastructure markets: The business of network slicing. In IEEE INFOCOM 2017-IEEE Conference on Computer Communications, pages 1-9. IEEE, 2017.

[18] A. Blogowski, P. Chrétienne, and F. Pascual. Network sharing by two mobile operators: beyond competition, cooperation. RAIRO-Operations Research, 49(3):635-650, 2015.

[19] A. Bousia, E. Kartsakli, A. Antonopoulos, L. Alonso, and C. Verikoukis. Game theoretic approach for switching off base stations in multioperator environments. In 2013 IEEE International Conference on Communications (ICC), pages 4420-4424. IEEE, 2013. 
[20] A. Bousia, E. Kartsakli, A. Antonopoulos, L. Alonso, and C. Verikoukis. Auction-based offloading for base station switching off in heterogeneous networks. In 2016 European Conference on Networks and Communications (EuCNC), pages 335-339. IEEE, 2016.

[21] A. Bousia, E. Kartsakli, A. Antonopoulos, L. Alonso, and C. Verikoukis. Game-theoretic infrastructure sharing in multioperator cellular networks. IEEE Transactions on Vehicular Technology, 65(5):3326-3341, 2016.

[22] A. Bousia, E. Kartsakli, A. Antonopoulos, L. Alonso, and C. Verikoukis. Multiobjective auction-based switching-off scheme in heterogeneous networks: To bid or not to bid? IEEE Transactions on Vehicular Technology, 65(11):9168-9180, 2016.

[23] C. Caillouet, D. Coudert, and A. Kodjo. Robust optimization in multi-operators microwave backhaul networks. In Global Information Infrastructure Symposium-GIIS 2013, pages 1-6. IEEE, 2013.

[24] L. Cano. Game-theoretic frameworks for the techno-economic aspects of infrastructure sharing in current and future mobile networks. PhD thesis, Polytechnique Montréal and Politecnico di Milano, 2020.

[25] L. Cano, A. Capone, G. Carello, M. Cesana, and M. Passacantando. Cooperative infrastructure and spectrum sharing in heterogeneous mobile networks. IEEE Journal on Selected Areas in Communications, 34(10):2617-2629, oct 2016.

[26] L. Cano, A. Capone, G. Carello, M. Cesana, and M. Passacantando. A non-cooperative game approach for RAN and spectrum sharing in mobile radio networks. In 22th European Wireless Conference, pages 1-6, Oulu, Finland, May 18-20, 2016.

[27] L. Cano, A. Capone, G. Carello, M. Cesana, and M. Passacantando. On optimal infrastructure sharing strategies in mobile radio networks. IEEE Transactions on Wireless Communications, 16(5):3003-3016, may 2017.

[28] L. Cano, G. Carello, M. Cesana, M. Passacantando, and B. Sansò. Modeling the technoeconomic interactions of infrastructure and service providers in $5 \mathrm{G}$ networks with a multi-leaderfollower game. IEEE Access, 7:162913-162940, dec 2019.

[29] C.-Y. Chang, N. Nikaein, O. Arouk, K. Katsalis, A. Ksentini, T. Turletti, and K. Samdanis. Slice orchestration for multi-service disaggregated ultra-dense RANs. IEEE Communications Magazine, 56(8):70-77, 2018.
[30] Z. Chang, D. Zhang, T. Hämäläinen, Z. Han, and T. Ristaniemi. Incentive mechanism for resource allocation in wireless virtualized networks with multiple infrastructure providers. IEEE Transactions on Mobile Computing, 19(1):103-115, 2018.

[31] Z. Chang, K. Zhu, Z. Zhou, and T. Ristaniemi. Service provisioning with multiple service providers in $5 \mathrm{~g}$ ultra-dense small cell networks. In IEEE PIMRC 2015, pages 1895-1900, 2015.

[32] X. Chen, H. Zhang, and Z. Han. Delay-tolerant resource scheduling in large-scale virtualized radio access networks. In 2017 IEEE International Conference on Communications (ICC), pages 1-6. IEEE, 2017.

[33] B. Cornaglia, G. Young, and A. Marchetta. Fixed access network sharing. Optical Fiber Technology, 26:2-11, 2015.

[34] X. Costa-Pérez, J. Swetina, T. Guo, R. Mahindra, and S. Rangarajan. Radio access network virtualization for future mobile carrier networks. IEEE Communications Magazine, 51(7):27-35, July 2013.

[35] S. Costanzo, I. Fajjari, N. Aitsaadi, and R. Langar. DEMO: SDN-based network slicing in CRAN. In 2018 15th IEEE Annual Consumer Communications $\&$ Networking Conference (CCNC), pages 1-2. IEEE, 2018.

[36] S. Costanzo, I. Fajjari, N. Aitsaadi, and R. Langar. A network slicing prototype for a flexible cloud radio access network. In 2018 15th IEEE Annual Consumer Communications \& Networking Conference (CCNC), pages 1-4. IEEE, 2018.

[37] S. Costanzo, D. Xenakis, N. Passas, and L. Merakos. Augmented RAN with SDN Orchestration of Multi-tenant Base Stations. Wireless Personal Communications, 96(2):2009-2037, 2017.

[38] L. A. DaSilva, J. Kibilda, P. DiFrancesco, T. K. Forde, and L. E. Doyle. Customized services over virtual wireless networks: The path towards networks without borders. In Future Network and Mobile Summit (FutureNetworkSummit), 2013, pages 1-10. IEEE, 2013.

[39] X. Deng, J. Wang, and J. Wang. How to Design a Common Telecom Infrastructure for Competitors to be Individually Rational and Collectively Optimal. IEEE Journal on Selected Areas in Communications, 35(3):736-750, 2017.

[40] P. Di Francesco, F. Malandrino, and L. A. DaSilva. Mobile network sharing between operators: a demand trace-driven study. In Proceedings of the 2014 ACM SIGCOMM workshop on Capacity sharing workshop, pages 39-44. ACM, 2014. 
[41] P. Di Francesco, F. Malandrino, T. K. Forde, and L. A. DaSilva. A sharing-and competition-aware framework for cellular network evolution planning. IEEE Transactions on Cognitive Communications and Networking, 1(2):230-243, June 2015.

[42] S. D'Oro, F. Restuccia, T. Melodia, and S. Palazzo. Low-complexity distributed radio access network slicing: Algorithms and experimental results. IEEE/ACM Transactions on Networking, 26(6):2815 - 2828, 2018.

[43] L. Doyle, J. Kibilda, T. K. Forde, and L. DaSilva. Spectrum without bounds, networks without borders. Proceedings of the IEEE, 102(3):351-365, 2014.

[44] S. E. Elayoubi, S. B. Jemaa, Z. Altman, and A. Galindo-Serrano. 5G RAN slicing for verticals: Enablers and challenges. IEEE Communications Magazine, 57(1):28-34, 2019.

[45] Ericsson. Network sharing. https://www.ericsson.com/us/ourportfolio/networksservices $/$ network-sharing? nav $=$ marketcategory004 [Online; Accessed: 2017-05-15].

[46] M. J. Farooq, H. Ghazzai, E. Yaacoub, A. Kadri, and M.-S. Alouini. Green virtualization for multiple collaborative cellular operators. IEEE Transactions on Cognitive Communications and Networking, 3(3):420-434, 2017.

[47] A. Fendt, S. Lohmuller, L. C. Schmelz, and B. Bauer. A network slice resource allocation and optimization model for end-to-end mobile networks. In 2018 IEEE $5 G$ World Forum (5GWF), pages 262-267. IEEE, 2018.

[48] R. Ferrus, O. Sallent, J. Pérez-Romero, and R. Agusti. On 5G radio access network slicing: Radio interface protocol features and configuration. IEEE Communications Magazine, 56(5):184-192, 2018.

[49] T. Frisanco, P. Tafertshofer, P. Lurin, and R. Ang. Infrastructure sharing for mobile network operators; From a deployment and operations view. In IEEE International Conference on Information Networking (ICOIN), pages 1-5, January 2008.

[50] L. Gao, P. Li, Z. Pan, N. Liu, and X. You. Virtualization framework and VCG based resource block allocation scheme for LTE virtualization. In 2016 IEEE 83rd Vehicular Technology Conference (VTC Spring), pages 1-6. IEEE, 2016.

[51] F. Grijpink, S. Newman, S. Sandoval, M. Strandell-Jansson, and W. Torfs. A "New Deal": Driving investment in Europe's telecoms infrastructure. https://tmt.mckinsey.com/content/industry/Telecommunications/page/19, 2012. [Online; Accessed: 2016-03-30].

[52] GSMA. Mobile infrastructure sharing. https://www.gsma.com/mobilefordevelopment/programme/connected-society/mobileinfrastructure-sharing-report/, November 2008. Accessed: 11-01-2019.

[53] A. Gudipati, L. E. Li, and S. Katti. RadioVisor: A slicing plane for Radio Access Networks. In Proceedings of the Third Workshop on Hot Topics in Software Defined Networking, HotSDN '14, pages 237-238, 2014.

[54] A. K. Gupta, J. G. Andrews, and R. W. Heath. On the feasibility of sharing spectrum licenses in mmwave cellular systems. IEEE Transactions on Communications, 64(9):3981-3995, 2016.

[55] J. Harno. 3G business case successfulness within the constraints set by competition, regulation and alternative technologies. JOURNAL-COMMUNICATIONS NETWORK, 1(2):159-165, 2002.

[56] J. He and W. Song. Appran: Application-oriented radio access network sharing in mobile networks. In Communications (ICC), 2015 IEEE International Conference on, pages 3788-3794. IEEE, 2015.

[57] S. L. Hew and L. B. White. Cooperative resource allocation games in shared networks: Symmetric and asymmetric fair bargaining models. IEEE Transactions on Wireless Communications, 7(11):4166-4175, November 2008.

[58] T. M. Ho, N. H. Tran, S. A. Kazmi, and C. S. Hong. Dynamic pricing for resource allocation in wireless network virtualization: A stackelberg game approach. In IEEE ICOIN 201\%, pages 429-434, 2017.

[59] T. M. Ho, N. H. Tran, L. B. Le, Z. Han, S. A. Kazmi, and C. S. Hong. Network virtualization with energy efficiency optimization for wireless heterogeneous networks. IEEE Transactions on Mobile Computing, 18(10):2386-2400, 2018.

[60] M. Hoffmann and M. Staufer. Network virtualization for future mobile networks: General architecture and applications. In 2011 IEEE international conference on communications workshops (ICC), pages 1-5. IEEE, 2011.

[61] M. F. Hossain, K. S. Munasinghe, and A. Jamalipour. Energy-efficient inter-RAN cooperation for non-collocated cell sites with base station selection and user association policies. Wireless Networks, 25(1):269-285, 2019. 
[62] F.-T. Hsu and C.-H. Gan. Resource allocation with spectrum aggregation for wireless virtual network embedding. In 2015 IEEE 82nd Vehicular Technology Conference (VTC2015-Fall), pages 1-5. IEEE, 2015.

[63] M. Hu, Y. Chang, Y. Sun, and H. Li. Dynamic slicing and scheduling for wireless network virtualization in downlink LTE system. In 2016 19th International Symposium on Wireless Personal Multimedia Communications (WPMC), pages 153-158. IEEE, 2016.

[64] J. Hultel, K. Johansson, and J. Markendahl. Business models and resource management for shared wireless networks. In IEEE 60th Vehicular Technology Conference (VTC2004-Fall), volume 5, pages 3393-3397, September 2004.

[65] Industry Canada. Framework for mandatory roaming and antenna tower and site sharing. http://www.ic.gc.ca/eic/site/smtgst.nsf/eng/ $\mathrm{h}_{s}$ f10290.html, 2013. [Online; Accessed : $2016-03-30]$.

[66] ITU. Mobile and wireless network regulation. http://www.ictregulationtoolkit.org/2.6. [Online; Accessed: 2016-03-28].

[67] T. Janssen, R. Litjens, and K. W. Sowerby. On the expiration date of spectrum sharing in mobile cellular networks. In 2014 12th International Symposium on Modeling and Optimization in Mobile, Ad Hoc, and Wireless Networks (WiOpt), pages 490-496. IEEE, 2014.

[68] Y. Jia, H. Tian, S. Fan, P. Zhao, and K. Zhao. Bankruptcy game based resource allocation algorithm for $5 \mathrm{G}$ Cloud-RAN slicing. In IEEE WCNC 2018, pages 1-6, 2018.

[69] K. Johansson. Cost effective deployment strategies for heterogeneous wireless networks. $\mathrm{PhD}$ thesis, KTH Information and Communication Technology, 2007.

[70] K. Johansson, M. Kristensson, and U. Schwarz. Radio resource management in roaming based multi-operator wcdma networks. In Vehicular Technology Conference, 2004. VTC 2004-Spring. 2004 IEEE 59th, volume 4, pages 2062-2066. IEEE, 2004.

[71] R. Jurdi, A. K. Gupta, J. G. Andrews, and R. W. Heath. Modeling infrastructure sharing in mmWave networks with shared spectrum licenses. IEEE Transactions on Cognitive Communications and Networking, 4(2):328-343, 2018.
[72] M. Kalil, M. Youssef, A. Shami, A. Al-Dweik, and S. Ali. Wireless resource virtualization: opportunities, challenges, and solutions. Wireless Communications and Mobile Computing, 16(16):2690-2699, 2016.

[73] M. I. Kamel, L. B. Le, and A. Girard. LTE wireless network virtualization: Dynamic slicing via flexible scheduling. In 2014 IEEE 80th Vehicular Technology Conference (VTC2014-Fall), pages 1-5. IEEE, 2014.

[74] E. Kapassa, M. Touloupou, P. Stavrianos, and D. Kyriazis. Dynamic $5 \mathrm{G}$ Slices for IoT applications with diverse requirements. In 2018 Fifth International Conference on Internet of Things: Systems, Management and Security, pages 195-199. IEEE, 2018.

[75] M. Katsigiannis, T. Smura, T. Casey, and A. Sorri. Techno-economic modeling of value network configurations for public wireless local area access. NETNOMICS: Economic Research and Electronic Networking, 14(1):27-46, November 2013.

[76] S. M. A. Kazmi and C. S. Hong. A matching game approach for resource allocation in wireless network virtualization. In Proceedings of the 11th International Conference on ubiquitous information management and communication, pages 1-6, 2017.

[77] K. J. Kerpez, J. M. Cioffi, P. J. Silverman, B. Cornaglia, and G. Young. Fixed access network sharing. IEEE Communications Standards Magazine, 1(1):82-89, 2017.

[78] M. A. Khan, A. C. Toker, C. Troung, F. Sivrikaya, and S. Albayrak. Cooperative game theoretic approach to integrated bandwidth sharing and allocation. In IEEE International Conference on Game Theory for Networks (GameNets '09), pages 1-9, May 2009.

[79] S. Khatibi and L. M. Correia. Modelling of virtual radio resource management for cellular heterogeneous access networks. In 2014 IEEE 25th Annual International Symposium on Personal, Indoor, and Mobile Radio Communication (PIMRC), pages 1152-1156. IEEE, 2014.

[80] J. Kibilda and L. A.DaSilva. Efficient coverage through inter-operator infrastructure sharing in mobile networks. In IEEE IFIP Wireless Days (WD), pages 1-6, November 2013.

[81] J. Kibiłda, N. J. Kaminski, and L. A. DaSilva. Radio access network and spectrum sharing in mobile networks: A stochastic geometry perspective. IEEE Transactions on Wireless Communications, 16(4):2562-2575, 2017. 
[82] J. Kibilda, F. Malandrino, and L. A. DaSilva. Incentives for infrastructure deployment by overthe-top service providers in a mobile network: A cooperative game theory model. In 2016 IEEE International Conference on Communications (ICC), pages 1-6. IEEE, 2016.

[83] W. Kiess, M. R. Sama, J. Varga, J. Prade, H.-J. Morper, and K. Hoffmann. 5G via evolved packet core slices: Costs and technology of early deployments. In 2017 IEEE 28th Annual International Symposium on Personal, Indoor, and Mobile Radio Communications (PIMRC), pages 1-7. IEEE, 2017.

[84] G. Koutitas, G. Iosifidis, B. Lannoo, M. Tahon, S. Verbrugge, P. Ziridis, Ł. Budzisz, M. Meo, M. A. Marsan, and L. Tassiulas. Greening the airwaves with collaborating mobile network operators. IEEE Transactions on Wireless Communications, 15(1):794-806, 2016.

[85] F. Kurtz, C. Bektas, N. Dorsch, and C. Wietfeld. Network slicing for critical communications in shared $5 \mathrm{G}$ infrastructures-an empirical evaluation. In 2018 4th IEEE Conference on Network Softwarization and Workshops (NetSoft), pages 393-399. IEEE, 2018.

[86] L.-V. Le, B.-S. P. Lin, L.-P. Tung, and D. Sinh. SDN/NFV, Machine Learning, and Big Data Driven Network Slicing for 5G. In 2018 IEEE $5 G$ World Forum (5GWF), pages 20-25. IEEE, 2018.

[87] H. Le Cadre and M. Bouhtou. An interconnection game between mobile network operators: Hidden information forecasting using expert advice fusion. Computer networks, 54(17):2913-2942, 2010.

[88] H. Le Cadre and M. Bouhtou. Modelling MNO and MVNO's dynamic interconnection relations: is cooperative content investment profitable for both providers? Telecommunication Systems, 51(2-3):193-217, 2012.

[89] T. LeAnh, N. H. Tran, D. T. Ngo, and C. S. Hong. Resource allocation for virtualized wireless networks with backhaul constraints. IEEE Communications Letters, 21(1):148-151, 2017.

[90] T. Levine, P. Eijsvoogel, and M. Reede. Passive infrastructure sharing. http://www.allenovery.com/SiteCollectionDocuments/Passive2012. [Online; Accessed: 2016-03-28].

[91] C. Liang and F. R. Yu. Wireless virtualization for next generation mobile cellular networks. IEEE wireless communications, 22(1):61-69, 2015.

[92] A. Lieto, I. Malanchini, S. Mandelli, E. Moro, and A. Capone. Strategic network slicing management in radio access networks. IEEE Transactions on Mobile Computing, 2020.

[93] P. Lin, J. Zhang, Q. Zhang, and M. Hamdi. Enabling the femtocells: A cooperation framework for mobile and fixed-line operators. IEEE Transactions on Wireless Communications, 12(1):158-167, 2013.

[94] Y.-T. Lin, H. Tembine, and K.-C. Chen. Interoperator spectrum sharing in future cellular systems. In Global Communications Conference (GLOBECOM), 2012 IEEE, pages 2597-2602. IEEE, 2012.

[95] Y. Lostanlen. From heterogeneous wireless networks to sustainable efficient ICT infrastructures. In 2013 rth European Conference on Antennas and Propagation (EuCAP), pages 1360-1363. IEEE, 2013.

[96] M. H. Lotfi and S. Sarkar. The economics of competition and cooperation between mnos and mvnos. In 201751 st Annual Conference on Information Sciences and Systems (CISS), pages 1-6. IEEE, 2017.

[97] X. Lu, K. Yang, Y. Liu, D. Zhou, and S. Liu. An elastic resource allocation algorithm enabling wireless network virtualization. Wireless Communications and Mobile Computing, 15(2):295-308, 2015.

[98] J. Lun and D. Grace. Software defined network for multi-tenancy resource sharing in backhaul networks. In 2015 IEEE Wireless Communications and Networking Conference Workshops (WCNCW), pages 1-5. IEEE, 2015.

[99] I. Malanchini, S. Valentin, and O. Aydin. Wireless resource sharing for multiple operators: Generalization, fairness, and the value of prediction. Computer Networks, 100:110-123, 2016.

[100] L. Mamatas, I. Psaras, and G. Pavlou. Incentives and algorithms for broadband access sharing. In Proceedings of the 2010 ACM SIGCOMM workshop on Home networks, pages 19-24, 2010.

[101] D. Marabissi and R. Fantacci. Heterogeneous public safety network architecture based on RAN slicing. IEEE Access, 5:24668-24677, 2017.

[102] J. Markendahl and M. Nilson. Business models for deployment and operation of femtocell networks; - Are new operation strategies needed for mobile operators? In 21st European Regional ITS Conference, Copenhagen, September 2010.

[103] M. A. Marsan and M. Meo. Network sharing and its energy benefits: A study of European mobile 
network operators. In 2013 IEEE Global Communications Conference (GLOBECOM), pages 2561-2567. IEEE, 2013.

[104] D.-E. Meddour, T. Rasheed, and Y. Gourhant. On the role of infrastructure sharing for mobile network operators in emerging markets. Computer Networks, 55(7):1576-1591, May 2011.

[105] O. Narmanlioglu and E. Zeydan. Efficient RRH assignments for mobile network operators in shared cellular network architecture. In 2017 IFIP/IEEE Symposium on Integrated Network and Service Management (IM), pages 1103-1108. IEEE, 2017.

[106] B. Naudts, M. Kind, F.-J. Westphal, S. Verbrugge, D. Colle, and M. Pickavet. Technoeconomic analysis of software defined networking as architecture for the virtualization of a mobile network. In 2012 European workshop on software defined networking, pages 67-72. IEEE, 2012.

[107] Nokia. White paper - network sharing: Delivering mobile broadband more efficiently and at lower cost. nokia.com. [Online; Accessed: 2017-05-15].

[108] F. Offergelt, F. Berkers, and G. Hendrix. If you can't beat 'em, join 'em; Cooperative and noncooperative games in network sharing. In IEEE 15th International Conference on Intelligence in Next Generation Networks (ICIN), pages 196-201, October 2011.

[109] F. H. Offergelt. Saphyre: Cooperation among competitors - analysing sharing scenarios for mobile network operators using game theory. Master's thesis, Leiden University, The Netherlands, 2011.

[110] M. Oikonomakou, A. Antonopoulos, L. Alonso, and C. Verikoukis. Cooperative base station switching off in multi-operator shared heterogeneous network. In 2015 IEEE Global Communications Conference (GLOBECOM), pages 1-6. IEEE, 2015.

[111] M. Oikonomakou, A. Antonopoulos, L. Alonso, and C. Verikoukis. Evaluating cost allocation imposed by cooperative switching off in multioperator shared hetnets. IEEE Transactions on Vehicular Technology, 66(12):11352-11365, 2017.

[112] M. Oikonomakou, A. Antonopoulos, L. Alonso, and C. Verikoukis. Fairness in multi-operator energy sharing. In 2017 IEEE International Conference on Communications (ICC), pages 1-6. IEEE, 2017.

[113] J. Ordonez-Lucena, P. Ameigeiras, D. Lopez, J. J. Ramos-Munoz, J. Lorca, and J. Folgueira. Network slicing for $5 \mathrm{G}$ with SDN/NFV: Concepts, architectures, and challenges. IEEE Communications Magazine, 55(5):80-87, 2017.

[114] J. S. Panchal, R. D. Yates, and M. M. Buddhikot. Mobile network resource sharing options: Performance comparisons. IEEE Transactions on Wireless Communications, 12(9):4470-4482, 2013.

[115] J. S. Park, M. Kim, and H. J. Lee. Analysis of European $3 \mathrm{G}$ markets and advanced strategies for $3 \mathrm{G}$ development. In The 7th International Conference on Advanced Communication Technology, 2005, ICACT 2005., volume 1, pages 428-431. IEEE, 2005.

[116] J. Pérez-Romero, O. Sallent, R. Ferrús, and R. Agustí. On the configuration of radio resource management in a sliced RAN. In NOMS 20182018 IEEE/IFIP Network Operations and Management Symposium, pages 1-6. IEEE, 2018.

[117] M. Rahman, C. Despins, and S. Affes. Analysis of CAPEX and OPEX benefits of wireless access virtualization. In IEEE International Conference on Communications (ICC) Workshops, pages 436-440, June 2013.

[118] P. Ramsdale. Personal communications in the UK-Implementation of PCN using DCS 1800. International Journal of Wireless Information Networks, 1(1):29-36, 1994.

[119] R. Ravindran, A. Chakraborti, S. O. Amin, A. Azgin, and G. Wang. 5G-ICN: Delivering ICN services over $5 \mathrm{G}$ using network slicing. IEEE Communications Magazine, 55(5):101-107, 2017.

[120] M. Rebato, M. Mezzavilla, S. Rangan, and M. Zorzi. Resource sharing in $5 \mathrm{~g}$ mmwave cellular networks. In Computer Communications Workshops (INFOCOM WKSHPS), 2016 IEEE Conference on, pages 271-276. IEEE, 2016.

[121] M. Richart, J. Baliosian, J. Serrati, J.-L. Gorricho, R. Agüero, and N. Agoulmine. Resource allocation for network slicing in WiFi access points. In 2017 13th International conference on network and service management (CNSM), pages 1-4. IEEE, 2017.

[122] R. Riggio, A. Bradai, D. Harutyunyan, T. Rasheed, and T. Ahmed. Scheduling wireless virtual networks functions. IEEE Transactions on network and service management, 13(2):240-252, 2016.

[123] B. Rouzbehani, L. M. Correia, and L. Caeiro. Radio resource and service orchestration for virtualised multi-tenant mobile Het-Nets. In 2018 IEEE Wireless Communications and Networking Conference (WCNC), pages 1-5. IEEE, 2018. 
[124] O. Sallent, J. Perez-Romero, R. Ferrus, and R. Agusti. On radio access network slicing from a radio resource management perspective. IEEE Wireless Communications, 24(5):166-174, 2017.

[125] K. Samdanis, X. Costa-Perez, and V. Sciancalepore. From network sharing to multi-tenancy: The $5 \mathrm{G}$ network slice broker. IEEE Communications Magazine, 54(7):32-39, 2016.

[126] T. Sanguanpuak, S. Guruacharya, E. Hossain, N. Rajatheva, and M. Latva-aho. Infrastructure sharing for mobile network operators: analysis of trade-offs and market. IEEE Transactions on Mobile Computing, 17(12):2804-2817, 2018.

[127] SAPHYRE. Deliverable D5.5, Business models, cost analysis and advices for spectrum policy and regulation for scenario III (full sharing), 2013.

[128] O. Semiari, W. Saad, M. Bennis, and Z. Dawy. Inter-operator resource management for millimeter wave multi-hop backhaul networks. IEEE Transactions on Wireless Communications, 16(8):5258-5272, 2017.

[129] M.-K. Shin, S. Lee, S. Lee, and D. Kim. A way forward for accommodating NFV in $3 \mathrm{GPP} 5 \mathrm{G}$ systems. In 2017 International Conference on Information and Communication Technology Convergence (ICTC), pages 114-116. IEEE, 2017.

[130] F. Shirzad and M. Ghaderi. Cloud-based spectrum sharing in virtual wireless networks. In 2016 IEEE 24th International Symposium on Modeling, Analysis and Simulation of Computer and Telecommunication Systems (MASCOTS), pages 196-204. IEEE, 2016.

[131] J. Simo-Reigadas, E. Municio, E. Morgado, E. M. Castro, A. Martinez, L. F. Solorzano, and I. Prieto-Egido. Sharing low-cost wireless infrastructures with telecommunications operators to bring $3 \mathrm{~g}$ services to rural communities. Computer Networks, 93:245-259, 2015.

[132] H. M. Soliman and A. Leon-Garcia. A novel neurooptimization method for multi-operator scheduling in cloud-RANs. In 2016 IEEE International Conference on Communications (ICC), pages 1-6. IEEE, 2016.

[133] H. M. Soliman and A. Leon-Garcia. QoSaware frequency-space network slicing and admission control for virtual wireless networks. In 2016 IEEE Global Communications Conference (GLOBECOM), pages 1-6. IEEE, 2016.

[134] F. Sun, B. Liu, F. Hou, H. Zhou, L. Gui, and J. Chen. Cournot equilibrium in the mobile virtual network operator oriented oligopoly offload- ing market. In 2016 IEEE International Conference on Communications (ICC), pages 1-6. IEEE, 2016.

[135] F. Teng, D. Guo, and M.-L. Honig. Sharing of unlicensed spectrum by strategic operators. In IEEE Global Conference for Signal Processing and Communications (GlobalSIP), pages 288-292, December 2014 .

[136] T. D. Tran and L. B. Le. Resource allocation for efficient bandwidth provisioning in virtualized wireless networks. In 2017 IEEE Wireless Communications and Networking Conference (WCNC), pages 1-6. IEEE, 2017.

[137] S. Valentin, W. Jamil, and O. Aydin. Extending generalized processor sharing for multi-operator scheduling in cellular networks. In Wireless Communications and Mobile Computing Conference (IWCMC), 2013 9th International, pages 485-490. IEEE, 2013.

[138] J. van de Belt, H. Ahmadi, L. E. Doyle, and O. Sallent. A prioritised traffic embedding mechanism enabling a public safety virtual operator. In 2015 IEEE 82nd Vehicular Technology Conference (VTC2015-Fall), pages 1-5. IEEE, 2015.

[139] F. Vaz, P. Sebastiao, L. Goncalves, and A. Correia. Femtocell deployment in LTE-A networks: A sustainability, economical and capacity analysis. In IEEE 24th International Symposium on Personal Indoor and Mobile Radio Communications (PIMRC), pages 3423-3427, September 2013.

[140] D. P. Venmani, Y. Gourhant, and D. Zeghlache. Divide and share: A new approach for optimizing backup resource allocation in LTE mobile networks backhaul. In 2012 8th International Conference on Network and Service management (CNSM) and 2012 Workshop on Systems Virtualization Management (SVM), pages 189-193. IEEE, 2012.

[141] D. P. Venmani, Y. Gourhant, and D. Zeghlache. ROFL: Restoration of failures through link-bandwidth sharing. In 2012 IEEE Globecom Workshops, pages 30-35. IEEE, 2012.

[142] J. Village, K. Worrall, and D. Crawford. 3g shared infrastructure. In Third International Conference on $3 G$ Mobile Communication Technologies (Conf. Publ. No. 489), pages 10-16. IET, 2002.

[143] M. Vincenzi, A. Antonopoulos, E. Kartsakli, J. Vardakas, L. Alonso, and C. Verikoukis. Cooperation incentives for multi-operator C-RAN energy efficient sharing. In 2017 IEEE International Conference on Communications (ICC), pages 1-6. IEEE, 2017. 
[144] P. L. Vo, M. N. Nguyen, T. A. Le, and N. H. Tran. Slicing the edge: Resource allocation for RAN network slicing. IEEE Wireless Communications Letters, 7(6):970-973, 2018.

[145] S. Wang, K. Samdanis, X. C. Perez, and M. Di Renzo. On spectrum and infrastructure sharing in multi-operator cellular networks. In 2016 23rd International Conference on Telecommunications (ICT), pages 1-4. IEEE, 2016.

[146] J. Wei, K. Yang, G. Zhang, and Z. Hu. Pricingbased power allocation in wireless network virtualization: A game approach. In 2015 International Wireless Communications and Mobile Computing Conference (IWCMC), pages 188-193. IEEE, 2015.

[147] J. Wei, K. Yang, G. Zhang, and X. Lu. A QoSAware Joint Power and Subchannel Allocation Algorithm for Mobile Network Virtualization. Wireless Personal Communications, 104(2):507-526, 2019.

[148] D. Wu, Z. Zhang, S. Wu, J. Yang, and R. Wang. Biologically inspired resource allocation for network slices in 5G-enabled Internet of Things. IEEE Internet of Things Journal, 6(6):9266-9279, 2018.

[149] Y. Xiao, Z. Han, C. Yuen, and L. A. DaSilva. Carrier aggregation between operators in next generation cellular networks: A stable roommate market. IEEE Transactions on Wireless Communications, 15(1):633-650, 2016.

[150] Y. Xiao, C. Yuen, P. Di Francesco, and L. A. DaSilva. Dynamic spectrum scheduling for carrier aggregation: A game theoretic approach. In Communications (ICC), 2013 IEEE International Conference on, pages 2672-2676. IEEE, 2013.

[151] D. Xu and Q. Li. Resource allocation in wireless virtualized networks with energy harvesting. In 2016 IEEE International Conference on Communication Systems (ICCS), pages 1-6. IEEE, 2016.

[152] Y. Zaki, L. Zhao, C. Goerg, and A. Timm-Giel. LTE mobile network virtualization: Exploiting multiplexing and multi-user diversity gain. Mobile Networks \& Applications, 16(4):424-432, August 2011.

[153] J. Zheng, P. Caballero, G. De Veciana, S. J. Baek, and A. Banchs. Statistical multiplexing and traffic shaping games for network slicing. IEEE/ACM Transactions on Networking, 26(6):2528-2541, 2018.

[154] R. Zhou, X. Yin, Z. Li, and C. Wu. Virtualized resource sharing in cloud radio access networks:
An auction approach. Computer Communications, 114:22-35, 2017.

[155] K. Zhu, Z. Cheng, B. Chen, and R. Wang. Wireless virtualization as a hierarchical combinatorial auction: An illustrative example. In 2017 IEEE Wireless Communications and Networking Conference (WCNC), pages 1-6. IEEE, 2017.

[156] K. Zhu and E. Hossain. Virtualization of 5g cellular networks as a hierarchical combinatorial auction. IEEE Transactions on Mobile Computing, 15(10):2640-2654, 2016. 


\section{AUTHORS}

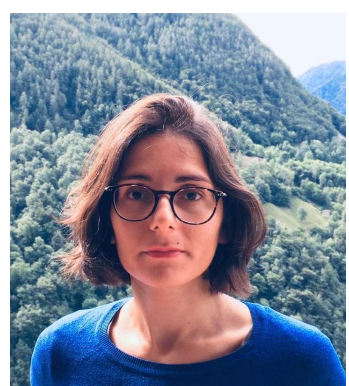

L. Cano (BSc. 12, MSc. 14, PhD 20) is currently a researcher at Politecnico di Milano. She recently obtained a double degree $\mathrm{PhD}$ at Politecnico di Milano and Polytechnique Montréal. Her main research interests are in the techno-economic characterization of infrastructure sharing in networks based on game theoretical models.

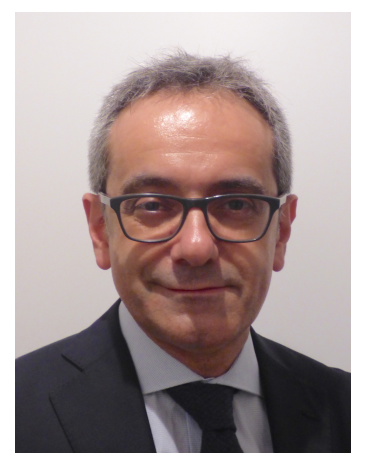

A. Capone (PhD 1998) is full professor at Politecnico di Milano where is he also the Dean of the School of Industrial and Information Engineering, member of the university strategy team POLIMI2040, and director of the Advanced Network Technologies Laboratory (ANTLab). He is associate editor with IEEE Trans. on Mobile Computing and Elsevier Computer Communications, and member of the TPC of major conferences in networking. His research interests include radio resource management and planning of wireless networks, software defined networks and switching architectures. On these topics he has published more than 300 papers. He is a fellow of the IEEE.

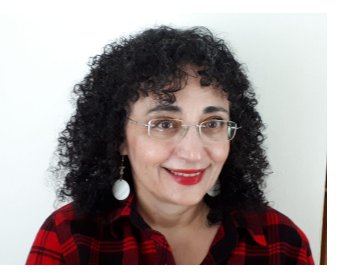

B. Sansò (Ph.D.89) is a full professor of telecommunication networks in the department of Electrical Engineering of Polytechnique Montréal and a member of GERAD, a world-renowned applied mathematics research center. Over her long career, she has received many awards and honors, has published and consulted extensively for industry and the mainstream media and has been part of major international committees. She leads the LORLAB, a research group dedicated to developing effective applied mathematics methods to the design and performance of wireless and wireline telecommunication networks. She has a special interest in network robustness and sustainability. 